\title{
Feasibility of utilizing the 200 -in. Hale Telescope as a deep-space optical receiver
}

\section{W. Tom Roberts, Hal L. Petrie, Andrew J. Pickles, Robert P. Thicksten, Chris Echols}

W. Tom Roberts, Hal L. Petrie, Andrew J. Pickles, Robert P. Thicksten, Chris Echols, "Feasibility of utilizing the 200-in. Hale Telescope as a deep-space optical receiver," Proc. SPIE 5550, Free-Space Laser Communications IV, (20 October 2004); doi: 10.1117/12.563324

SPIE Event: Optical Science and Technology, the SPIE 49th Annual Meeting, 2004, Denver, Colorado, United States 


\title{
Feasibility of utilizing the 200-inch Hale telescope as a deep-space optical receiver
}

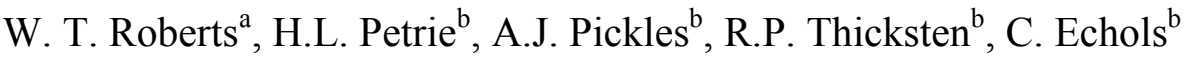 \\ ${ }^{\mathrm{a}}$ Jet Propulsion Laboratory, California Institute of Technology, 4800 Oak Grove Drive, Pasadena, \\ CA 91109; \\ ${ }^{\mathrm{b}}$ Caltech Optical Observatories, California Institute of Technology, 1201 E. California Blvd, \\ Pasadena, CA 91125.
}

\begin{abstract}
Capturing the very faint optical communications signals expected from the Mars Laser Communication Demonstration (MLCD) experiment to fly aboard the Mars Telecommunications Orbiter (MTO) in 2009 requires a sensitive receiver placed at the focus of a large collecting aperture. For the purpose of demonstrating the potential of deep-space optical communication, it makes sense to employ a large astronomical telescope as a temporary receiver. Because of its large collecting aperture, its reputation as a well-run instrument, and its relative convenience, the 200-inch Hale Telescope on Palomar Mountain is being considered as a demonstration optical 'antenna' for the experiment. However, use of the telescope in this manner presents unique challenges to be overcome, the greatest of which is pointing the telescope and maintaining the communication link to within a few degrees of the Sun. This paper presents our candidate approaches for adapting the Hale telescope to meet the demonstration requirements, modifications to the facilities and infrastructure, the derivation of requirements for baffles and filters to meet the near-Sun pointing objectives, and initial data on the potential of candidate modifications to meet the requirements.
\end{abstract}

\subsection{INTRODUCTION}

The Mars Laser Communication Demonstration (MLCD) project is an experiment slated to fly on the Mars Telecommunication Orbiter (MTO) in 2009. The principal objectives of the project are to demonstrate the capabilities and efficacy of high-bandwidth laser communications from Mars to Earth in support of future deep space exploration and discovery missions ${ }^{1,2}$.

In order to concentrate resources on development of the challenging flight terminal, the project is considering the use of existing ground-based telescopes for collecting the extremely faint signal from the spacecraft's 5-Watt laser transmit terminal. With only a few photons per square meter incident at the ground from the greatest distances, large, efficient collecting apertures are required to achieve the megabit-per-second (Mbps) requirements. Maximizing the available communication time requires the placement of the receive terminal at a site with good weather statistics, and minimization of atmospheric transmission loss and atmospheric scatter drive the selection of relatively high-altitude sites. Finally, the amount of collected background noise is dependent on the field of view the data detector must cover, which drives the selection of a ground terminal receiver site to a location with good atmospheric seeing. These criteria all support the model of using astronomical telescopes as interim ground-based receivers.

One of the main concerns of deep-space communication links is the outage period occurring when the solar-system geometry requires that one or both of the receive terminals must point near the Sun. In some cases, the Sun may actually physically interrupt the line-of-sight between the terminals, though more generally there is some angular zone around the Sun within which the terminal cannot point or effectively communicate. The orbital geometry then dictates the frequency of such outages, and the angular exclusion zone determines their duration. As an example, for the 2011 MarsSun conjunction, exclusion angles (Figure 1) of 30 degrees, 10 degrees, 5 degrees and 3 degrees result in outage periods of 256 days, 89 days, 44 days and 27 days respectively. 


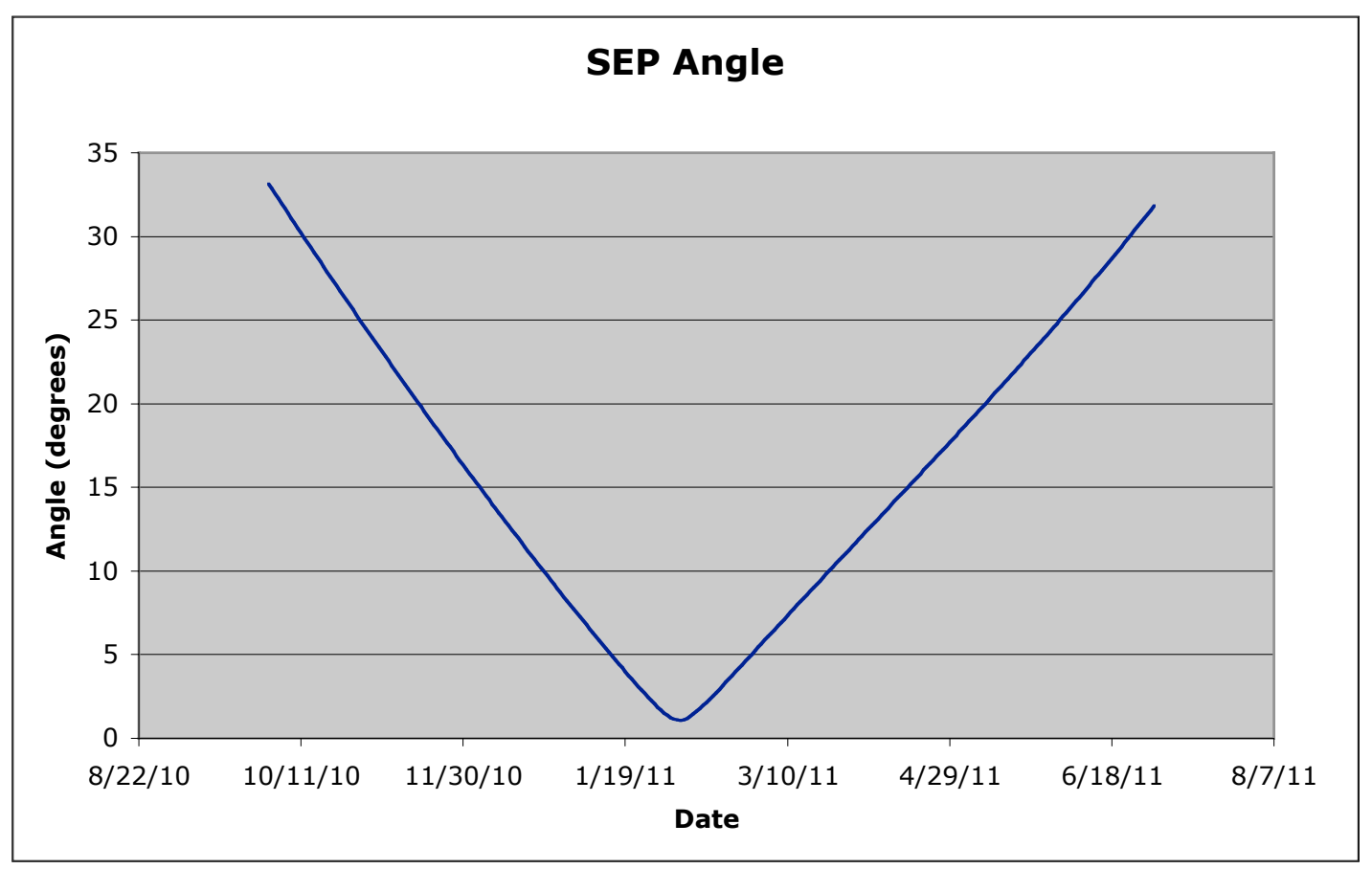

Figure 1 Sun-Earth-Probe (SEP) angle for Mars 2010-2011 solar conjunction.

To be a useful high-bandwidth communication link, it is very desirable to limit the solar exclusion time to about one month, resulting in an exclusion angle of about 3 degrees. This goal comprises the most stressing technical requirement for accomplishing the receiver task using astronomical telescopes.

\subsection{DAYTIME USE OF TELESCOPES}

The problems associated with the daytime use of telescopes*, especially while pointing close to the Sun, can be divided into three categories based on the severity of the issue. The most benign of these issues are those that degrade or inhibit performance of the laser communication demonstration, and can generally be handled totally internally to the project. Background radiance of the sky and planet, scattered light at the focal plane, seeing from any removable objects in the optical system, signal polarization and transmission effects all fall under this category.

Of somewhat more concern are those effects that will have a short-term impact on the use and performance of the telescope. Items in this category include seeing effects resulting from localized heating of the dome, telescope and structure, and temporary recoverable optical degradation resulting from figure changes to optical surfaces and misalignment of surfaces due to differential heating of the telescope structure. Such affects will hinder not only the MLCD operations, but also any immediate subsequent observers; thus, it is a matter of concern to all telescope parties, and should be addressed cooperatively by the MLCD project and Caltech Optical Observatories. Since this category is comprised only of relatively short-term thermal effects, the simplest approach to addressing these effects is to allow extra time (typically 1-2 additional days) for the telescope and structures to cool and come to thermal equilibrium. This is somewhat objectionable from an efficiency standpoint, since it is wasting valuable time on one of the world's great astronomical telescopes. More palatable is the concept of scheduling less resolution-oriented experiments and events immediately after MLCD observational periods. Examples of this are certain types of spectroscopy measurements, engineering experiments, and maintenance down-time. Finally, more aggressive alterations to the telescope and its facilities might be undertaken to alleviate this category of problems. Such alterations include improved and/or increased air handling and ventilation for exchange of warm dome air with cooler external air, increased air conditioning for active

\footnotetext{
* The use of the word telescope will imply astronomical telescope throughout this paper. Solar telescopes have been considered for this task, but because of the severe aperture limitation of existing solar telescopes, cannot meet MLCD data rate requirements.
} 
cooling of the inside of the dome, and active support for the primary mirror to counteract any thermal distortions resulting from long-term daytime operations.

By far the most important of the three categories of effects is the final category, defined as those effects which threaten the long-term quality of the instrument, or safety of the instrument, facilities and personnel. This category includes the potential of significant differential heating of the primary mirror to exacerbate the propagation of existing minor mirror cracks, and all of the various undesirable scenarios which might result from the focused primary-mirror-generated image of the Sun. The irradiance of this focused image is, to first order, given by the camera equation

$$
E=\frac{\pi L}{4 F^{2}}
$$

in which $\mathrm{L}$ is the solar radiance, and $\mathrm{F}$ is the focal ratio of the primary mirror. This results in irradiance values at focus of up to $100 \mathrm{~W} / \mathrm{cm}^{2}$, or about 1000 times the full solar intensity at the surface of the earth. Such intensities have the potential for causing permanent thermal damage to the structure, harming personnel who may be near the focused image, or starting a fire within the dome. The significance of the impact of such failures drives the initial approach to solving the problems associated with the use of the telescope, and acts as a mandatory hurdle which must be overcome to proceed to design. If these problems cannot effectively and reliably be addressed, there is no need continuing with the concept.

\subsection{Dome Slit Shield}

\subsection{SOLUTION APPROACH}

Though dome heating is not a serious concern for protecting the health of the telescope and facilities, it is nevertheless of great importance in trying to make efficient use of telescope time, or even expand the number of hours the telescope might be used. Several options have been considered for minimizing the seeing effects resulting from increased heat loading inside the telescope dome. An obvious course of action is to paint surfaces with a white paint that is highly reflective in the visible, but with high emissivity in the thermal infrared (8-12 micron wavelengths). There are significant concerns that this can work too well on the upper surfaces of the dome and telescope structures, such that surfaces cool below the dewpoint at night, allowing water to condense out and drip onto the primary mirror. It has also been suggested that liberal use of insulation can prevent heat buildup in structures that might later contribute significantly to dome seeing. Examples of this might be construction of removable multilayer insulation (MLI) or packing foam shields for the upper telescope structures, and packing foam disks that could be distributed across the dome floor during daytime operations. Although these devices would reflect most of the energy away from thermally massive structures, the limited dome slit opening would still retain most of the heat in the dome, resulting in a thermal boundary between the dome air and external air. As a result, it is expected that there would still be significant seeing degradation.

It is preferred to limit the energy admitted to the dome as much as possible. The first line of defense should thus be at the dome slit; it should be necessary only to have a circular opening slightly larger than the 5-meter entrance pupil of the telescope, and seal off the rest of the dome from incoming solar radiation. The daily solar power flux is dependent on the dome opening, the season, and the SEP angle. To estimate the savings in dome heat which must eventually be expelled, four dates during the hypothetical baseline mission in the 2010-2011 operational time period were evaluated. For each of these dates, the total amount of flux through the fully-open dome slit and through a 6-meter diameter aperture were calculated at 1-hour intervals. In each calculation, it was assumed that the telescope was pointed directly at the spacecraft, and the dome slit was centered to the telescope azimuth.

The relative amounts of power throughout a daytime pass are shown for these representative dates in Figure 2 . In general, the total integrated power is reduced by almost an order of magnitude by implementing the dome shield. The curves for 15 June, 2010 assumes an SEP angle of 72 degrees, and has the spacecraft in cruise to Mars, rising above 20 degrees of elevation around noon. In this case, the dome is opened to the northeast at approximately noon, allowing a significant influx of light from the high Sun. As the Sun rapidly descends toward the west, the flux into the dome rapidly falls off. Because of the projected angle of the 6-meter aperture case, the amount of energy entering the dome daily through this aperture is only about $5 \%$ of the amount that would enter without the dome shield. During passes with lower SEP angles, integrated fluxes are reduced to about $11-13 \%$ of those nominally incident. Peak fluxes are observed for the pass of 15 Feb, 2011, when Mars is only 2.3 degrees from the Sun. During this pass the 6-meter aperture is held 


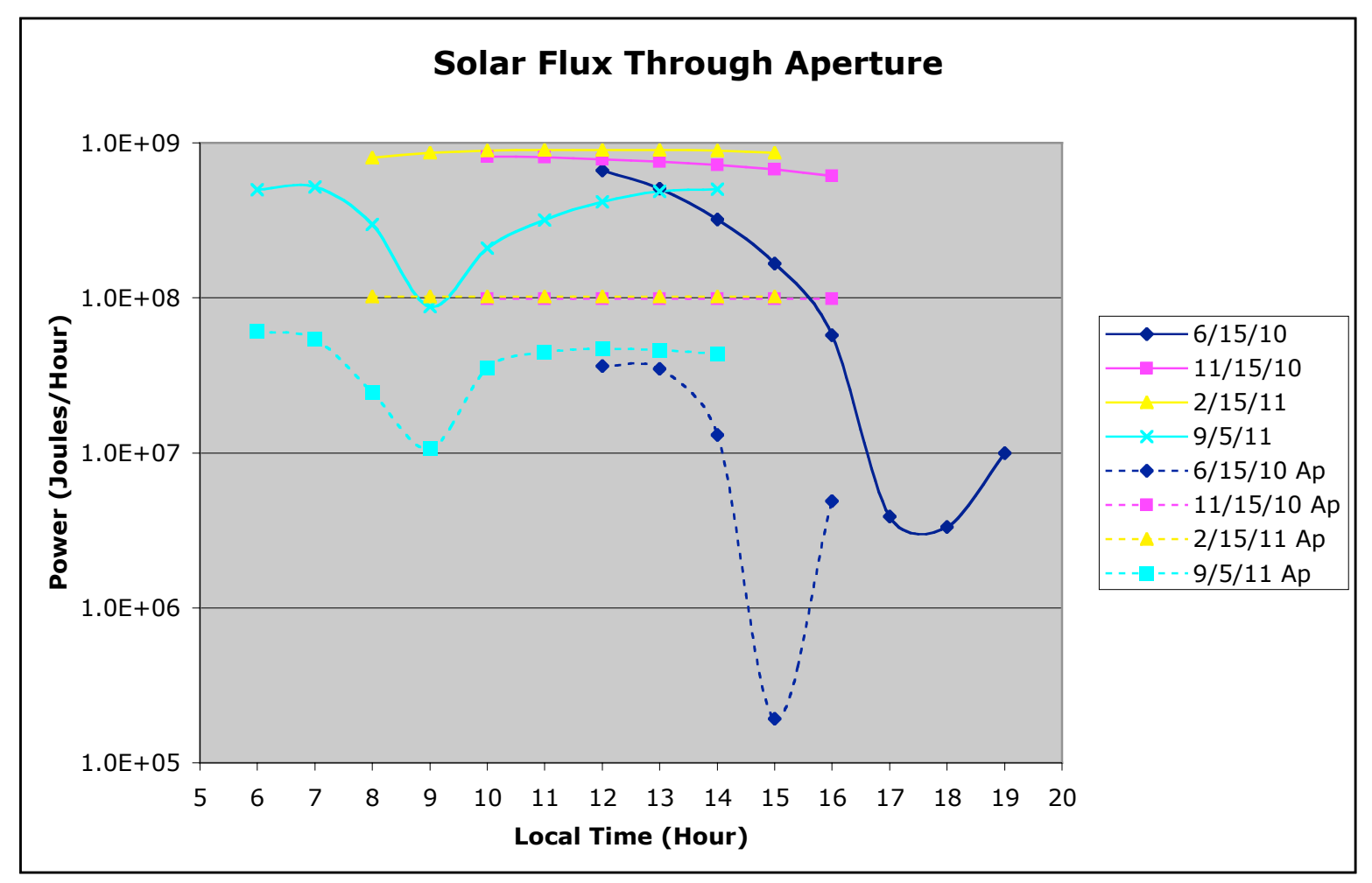

Figure 2. Solar flux into the fully open dome slit (solid lines) and through a 6-meter aperture (broken lines) for various mission days.

practically normal to the Sun throughout the pass, resulting in a constant power during the pass. The uneven curves associated with 15 September, 2011 are the result of the relatively large 54-degree SEP angle. During local morning and evening, the dome slit is aligned with the ecliptic, so sunlight directly enters the dome. Around 9:00 AM Mars has transitioned to the western sky, while the Sun is still in the east, resulting in a minimum in the flux curve. As the Sun comes around to the western sky, the flux in the dome gradually increases until the dome slit is once again aligned with the ecliptic.

The basic structure for some of the dome shield already exists in the form of the Hale Telescope's wind screen, a canvas screen that is drawn up from the bottom of the slit to limit wind in the dome. The screen is suspended from bars that run the width of the slit, and run in a track along the inside of the dome slit from below the telescope to an elevation angle of 70 degrees. To complete a dome shield, it would be necessary to install two additional sets of track alongside the existing track. One set of tracks would extend from the top of the dome slit down to the bottom for a similar canvas screen which unfurls from the top. The other additional track would also extend from the top of the telescope to the bottom, and would contain a solid-frame shield with the required cut-out aperture. A reflective outer surface should be applied to the canvas screens to avoid heat absorption for possible transfer to the inside of the dome.

Because of the high payoff and relative simplicity of the dome slit shield, and because, once installed, it can be rolled into place or stowed during astronomical use of the telescope, this is one of the most attractive heat-mitigation techniques. It appears that this is a feasible option, and there appear to be no drawbacks to its implementation. It has therefore been adopted as a baseline approach.

\subsection{Baffles}

The approach proceeds by addressing the third category of problems listed above, all of which are the result of direct solar illumination of the primary mirror. The most effective and generally the least expensive way to eliminate this is through baffling. The first level of baffling is that provided by the telescope dome. By opening the dome slit only 
partially such that the width of the opening is only slightly larger than the diameter of the entrance pupil, it should be possible to point the telescope to within 12 degrees of the Sun without directly illuminating the primary mirror, as shown in Figure 3. At 3 degrees, over $50 \%$ of the primary mirror would be illuminated (after accounting for the shadow of the secondary and spider obscuration), forcing the installation of additional baffling.

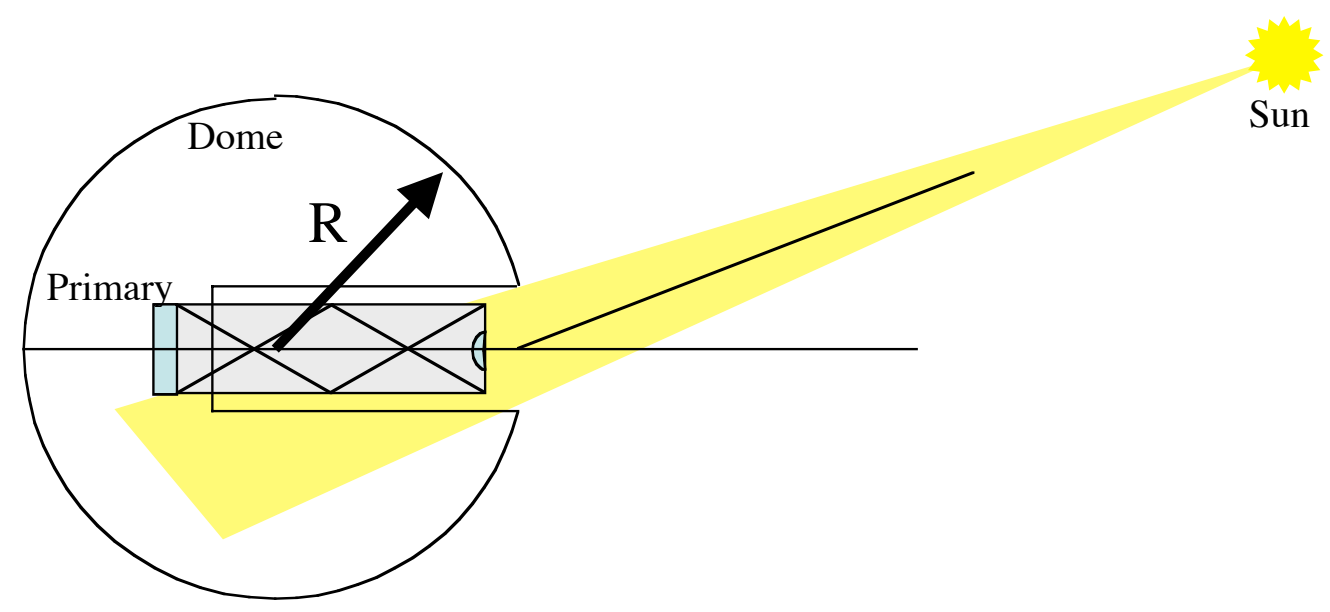

Figure 3 Diagram showing use of a telescope dome to occult the telescope primary mirror from sunlight.

To get to 3 degrees, an aspect ratio (baffle length to wall separation ratio) of roughly 20:1 is required. A mock test baffle was constructed for testing at 3 degrees by painting a large honeycomb baffle with Cat-a-lac black. The honeycomb cells were $1 \mathrm{inch}$ wide and 20 inches long, to meet the aspect ratio requirements. Initial testing of the baffle demonstrated that while this design would be sufficient to completely prevent any direct sunlight from illuminating the primary mirror, it would introduce far too much scattered light for near-Sun operations. Figure 4 shows an outdoor demonstration of the baffle mock-up, where the baffle is held such that its axis is pointed 3 degrees from the Sun, and the photograph is taken along the baffle optical axis. In the photo, the Sun is obscured by the edge of the building. This photograph demonstrates that the baffle-induced irradiance at the focal plane dominates the sky background. Clearly, a more careful, systematic development of a baffle would be required to allow operation of the baffle system at low angles. Tapered baffles and inverted-T baffles were considered, but the problems associated with fabricating and maintaining alignment, and increasing obscuration made this approach less and less attractive.

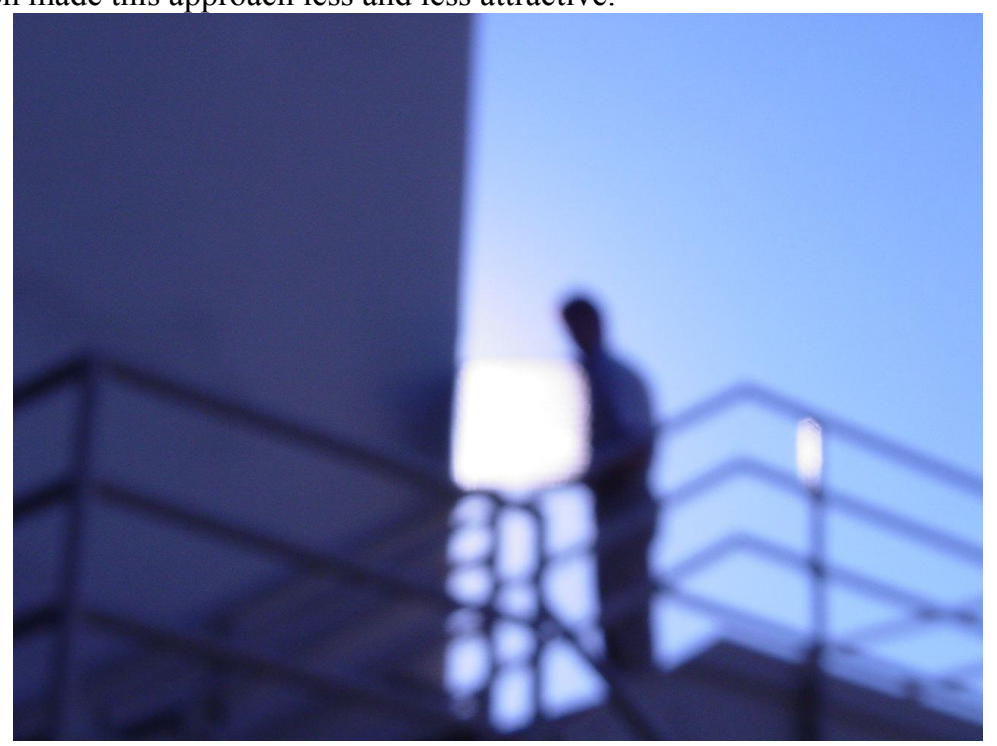

Figure 4. Honeycomb baffle reflecting sunlight at 3 degrees. Scatter from baffle is brighter than atmospheric scatter. 
A second problem with the baffle is that in absorbing the full amount of incident sunlight, the baffle surfaces must necessarily warm. Tests of similar baffles exposed to sunlight demonstrated a warming to over 30 degrees $\mathrm{C}$ above ambient temperature in breezy conditions. The dominant mechanism for shedding the heat, at least in simple designs, is through convection to the local atmosphere. This was predicted to worsen the seeing considerably, again forcing the collection of too much background to maintain communications.

\subsection{Filters}

The second approach to limiting the off-axis sunlight incident on the primary mirror is the introduction of a spectral filter in the field of view of the telescope. Such a filter must be designed to efficiently transmit the communication laser wavelength, while rejecting the majority of the remainder of the solar spectrum. To avoid induced-seeing problems already encountered during the baffle development, it is preferable that the filter be designed to reflect the out-of-band solar radiation, rather than absorb it. Using multi-layer interference coatings this is possible, at least on smaller filter segments. The challenge will be to make, support and align such a filter large enough to protect the entire 5-meter aperture of the telescope, without interfering with the received signal.

A second concern of a filter is the introduction of another scattering surface in the telescope field of view. Because the filter will be directly exposed to full sunlight, it has the potential for generating a large amount of forward scatter into the detector field of view. This scatter would occur across the entire solar spectrum, but most importantly in the pass-band of the filter. To maintain high-rate operation at near-conjunction times, a scattering budget of approximately $0.03 \mathrm{sr}^{-1}$ has been levied for scatter from the solar filter. This budget includes scatter from surface roughness, internal substrate scattering and environmental contamination. Though optical surfaces much better than this are standard, obtaining this quality and maintaining cleanliness over a large surface is considered challenging.

\subsection{FILTER REQUIREMENTS}

Most of the filter requirements for protection from dome-heating and seeing effects are easily derived from first-order analysis of dome flux, similar to the calculations performed above. However, it was deemed that a much more detailed thermal model was required to investigate the thermal effects on the Hale Telescope primary mirror. In part, this was to estimate the temperature rise expected from low level, long-duration exposure to filtered sunlight to estimate the primary mirror aberration and residual seeing effects that might result over the next few hours. More important, however, was the need to assess the internal stresses that might result in the primary mirror, and evaluate the potential of these stresses to extend or deepen the existing cracks in the primary mirror.

\subsection{Primary Mirror Modeling}

A solid CAD model of the P200 primary mirror was constructed in SolidWorks. The waffle pattern of the mirror's glass was imported from AutoCAD and extruded up to a 5 inch thick curved mirror surface with a radius of curvature of 1332 inches. This resulted in a very realistic solid model of the mirror, shown below in Figure 5. An area of 6544.2 square inches $\left(4.2 \mathrm{~m}^{2}\right)$, representing the occultation of the Sun upon the mirror, was constructed inside an arc on one side of the mirror, as shown in the Figure.

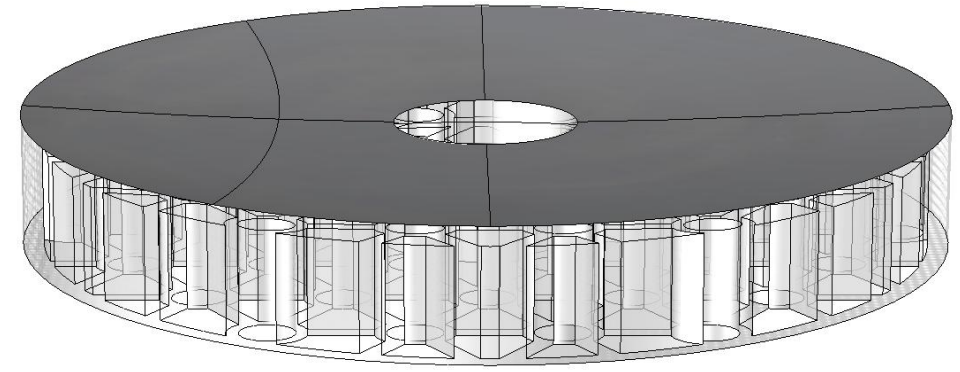

Figure 5 Solid model of Hale Telescope primary mirror. Arc on left delineates sunlit area. 
Using COSMOSWorks, a solid mesh was constructed utilizing 96771 total nodes and 50608 total elements. The global element size was set at 4 inches, with a tolerance of .2 inches. A thermal analysis was performed, in which a convection boundary condition of $2 \mathrm{~W} / \mathrm{m}^{2}$ to a $283 \mathrm{~K}$ atmosphere was applied to the mirror surface. A heat flux of $5.4 \mathrm{~W} / \mathrm{m}^{2} \mathrm{was}$ applied to the Sun exposure area, based on $1353 \mathrm{~W} / \mathrm{m}^{2}$ incident solar power, $5 \%$ transmission through the solar filter, and $8 \%$ absorption in the aluminum coating of the primary. This input was assumed to exist continuously for the duration of an 8-hour pass. The results of the thermal run are shown in Figure 6 . The maximum temperature rise is predicted to be $0.59^{\circ} \mathrm{C}$.

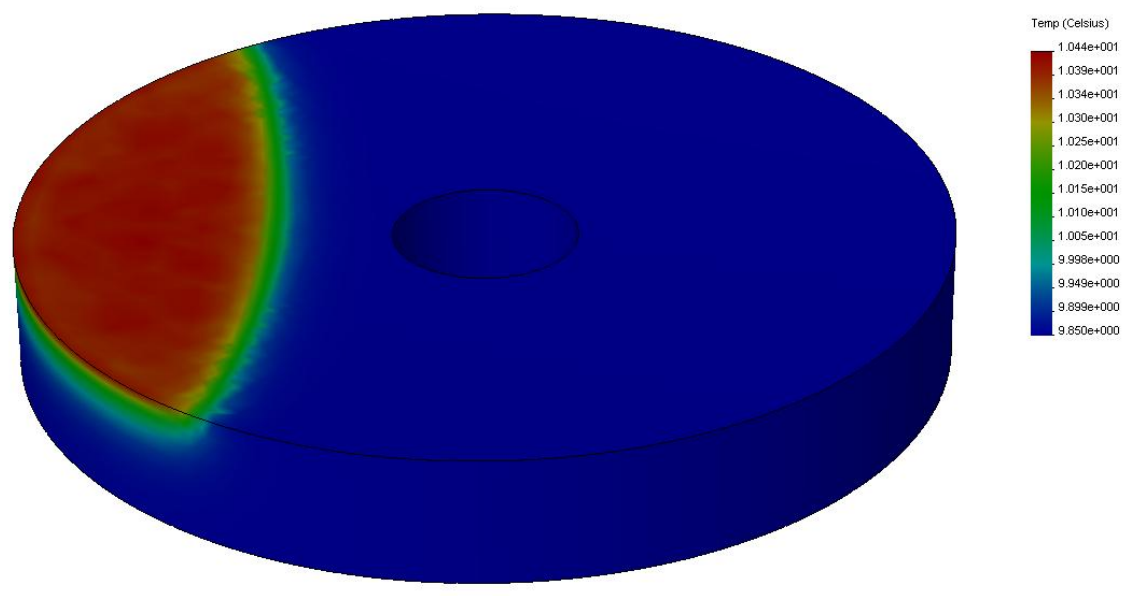

Figure 6 Temperature plot showing temperature distribution after 8 hours of $5.4 \mathrm{~W} / \mathrm{m}^{2}$ input to the heated area on left.

Following the thermal analysis, a stress analysis was performed based on the thermal results. The maximum resultant displacement, mostly at the edge of the mirror, was predicted to be about $2 \mathrm{~mm}$. This results in internal stress levels up to $76 \mathrm{kPa}$ (11 PSI), as shown in Figure 7 below. This level is well below the 100 PSI limit placed to avoid exacerbating the mirror cracks from the original mirror annealing in the late 1930's.

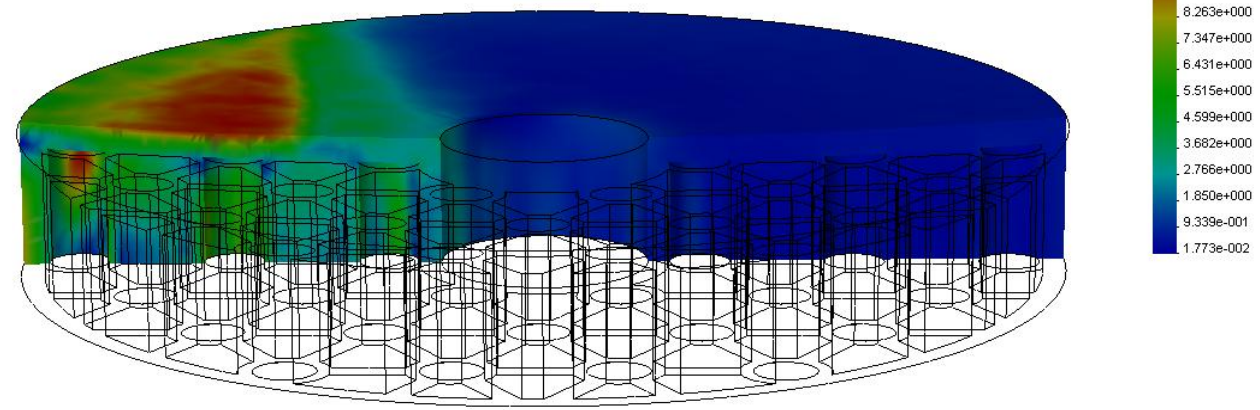

Figure 7. Thermally induced stresses in the Hale Telescope primary mirror after 8 hours of exposure to a $5.4 \mathrm{~W} / \mathrm{m} 2 \mathrm{input}$ on the left. Maximum stress is $76 \mathrm{kPa}$. 


\subsection{Thermal Tests}

To investigate unanticipated thermal problems, and demonstrate the ability to expose the telescope and the dome interior to full, direct solar radiation, an experiment was performed on the mornings of April 13 and 14, in which the dome was opened and the telescope pointed directly at the Sun for a period of 15 minutes. To eliminate the possibility of catastrophic damage from primary mirror illumination, the primary mirror cover was closed during the experiment. Various locations around the dome, on the primary mirror, and on the telescope structure were outfitted with thermal sensors. The sensors were left in place and the various temperatures recorded over a period of several days prior to the exposure to establish a baseline expected excursion. Temperature sensors placed around the top ring of the telescope saw the greatest perturbations, and are shown in Figure 8. Temperature rises of up to 20 degrees Fahrenheit $(11 \mathrm{C})$ were observed on the aluminum tape-covered steel prime-focus ring directly facing the Sun. Moreover, the temperature rise is continuing unabated at the end of the 15-minute exposure, indicating the potential for much higher temperatures during a long exposure period. Temperatures on the head ring recover to the nominal daytime thermal profile within approximately 3 hours of closing the dome. During this experiment, the dome was held open to full solar flux for 15 minutes. During this time, the amount of heat entering the dome was approximately equal to the amount entering the dome through the open dome-shield aperture over a 2 hour period. Assuming a 5\% spectrally transmitting solar filter fills the dome-shield aperture, the amount of energy accumulated during this experiment greatly exceeds the amount of energy that would enter the dome during a single pass.

Temperatures - P.F. Ring Area 04/12/04 -- 04/14/04

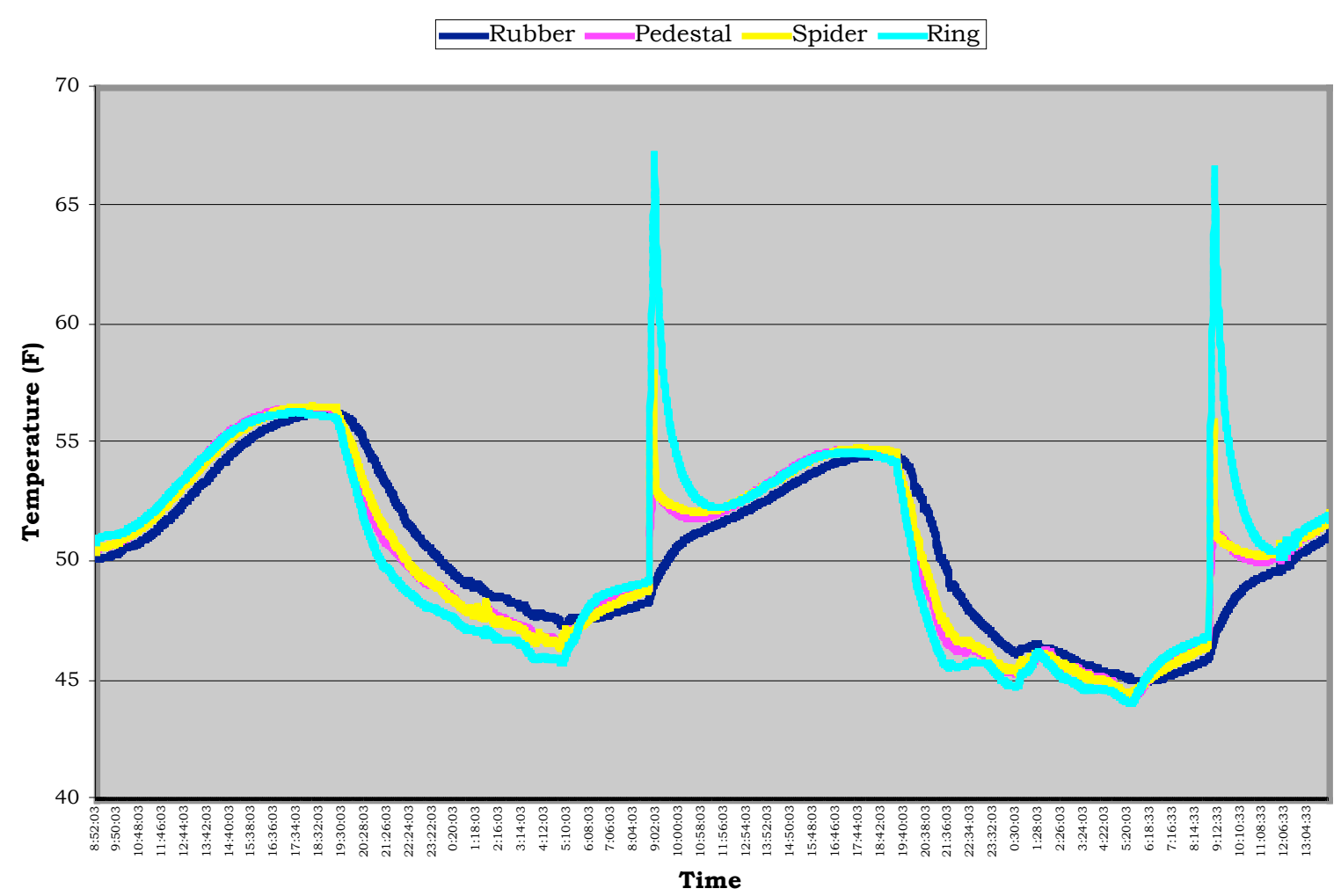

Figure 8. Measurements of temperature at selected locations on the prime focus ring. Spikes indicate temperature rise during 15minute exposure to direct, unfiltered sunlight.

\subsection{Filter Requirements}

The requirements for the solar protection filter are shown in Table 1 below. These performance specifications tend to be somewhat conservative. They were derived based on the analyses listed above, and simple optical performance calculations. As the design of the receiver matures, the performance ranges are likely to shift slightly, resulting in potential cost savings. 
Table 1 Filter requirements for Palomar Solar Protection Filter

\begin{tabular}{|l|l|}
\hline Parameter & Specification \\
\hline Shape/Size & Equilateral hexagon with side length of at least 0.25 meters \\
\hline Thickness & $0.3-1.0 \mathrm{~cm}$ depending on size \\
\hline Transmission & $90 \%$ external transmission from $1059.0 \mathrm{~nm}$ to $1061.0 \mathrm{~nm}$ \\
\hline Incident Angle & $0.0+/-1.0$ degree \\
\hline Blocking & $95 \%$ to $98 \%$ of the complete incident solar power spectrum \\
\hline Uniformity & All specifications must be met averaged over entire filter area \\
\hline Polarization & Non-polarizing to within $1 \%$ at normal incidence \\
\hline Absorption & Absorbs less than $10 \%$ of incident solar power \\
\hline Flatness & $\lambda / 4$ at 1060 nm \\
\hline Scatter & BSDF at 3 degrees from normal $<0.03 \mathrm{sr}^{-1}$ at $1060 \mathrm{~nm}$ \\
\hline Wedge & $<1$ second of arc \\
\hline Operating Temp & $-10 \mathrm{C}$ to $+80 \mathrm{C}$ \\
\hline
\end{tabular}

At this stage, there is some flexibility in the design; for example, it may be advantageous to employ larger segments, which will generate less obscuration to the incoming beam from reduced framework. The number of segments required to cover the full aperture, and the frame length and fractional obscuration for various sized segments are shown in Table 2 below. In this table, the side length is the integrated length of the frame visible in the entrance pupil of the telescope which will result in the indicated obscuration.

Table 2 Hexagon parameters for filling Hale Telescope aperture

\begin{tabular}{|l|l|l|l|l|}
\hline & $\mathbf{2}$ meter & $\mathbf{1 . 2}$ meter & $\mathbf{0 . 8 5}$ meter & $\mathbf{0 . 5 5}$ meter \\
\hline Number of Segments & 7 & 19 & 37 & 91 \\
\hline Side Length (m) & 20 & 33 & 46 & 59 \\
\hline Fractional Obscuration (\%) & 2.3 & 3.7 & 5.2 & 8.1 \\
\hline
\end{tabular}

\subsection{SUMMARY AND CONCLUSIONS}

It appears feasible for the 200-inch Hale Telescope on Palomar Mountain to be converted into an optical receiver for the MLCD experiment. The main modifications that would be required are to the dome; a dome slit shield with an aperture of just over 5 meters will limit the flux of solar light onto the primary mirror at SEP angles down to about 12 degrees, and will reduce the total heat flux into the dome by almost an order of magnitude. This dome shield then becomes a very attractive location for a spectral filter for reflecting away most of the Sun's light, while efficiently admitting the communication laser wavelengths. This solar filter can reasonably be designed with multilayer coatings to reflect away $95-98 \%$ of the incident sunlight, resulting in greatly reduced amounts of energy being absorbed in the primary mirror or being brought to focus on structural elements near the prime focus cage. The combination of the dome slit shield and spectral filter should limit the energy into the dome to about 2-5 x $10^{7}$ Joules/day for the worst days (near SEP minimum). Active mirror control and improved ventilation are currently being considered to alleviate any residual effects from daytime operations. Since the majority of Mars-to-Earth optical communications will occur during daylight hours, it is hoped that the overall effects on the telescope will be benign to the degree that concurrent operations between daytime communications and nighttime astronomy can be achieved.

\section{ACKNOWLEDGEMENTS}

The authors would like to thank Dr. Gary Peterson of Breault Research Organization for calculations indicating relative amounts of power into dome slit. The Jet Propulsion Laboratory and California Institute of Technology carried out the research described in this paper under contract with the National Aeronautics and Space Administration. 


\section{REFERENCES}

1. Bernard. L. Edwards, Stephen A. Townes, Roy S. Bondurant, Joseph J. Scozzafava, Don M. Boroson, William T. Roberts, Abhijit Biswas, Alen D. Pillsbury, Farzana I. Khatri, Jamie W. Burnside, David R. Bold, Daniel V. Murphy, Alexander K. McIntosh, David O. Caplan, Alan E. DeCew, Jayant Sharma, Ben A. Parvin, Richard J. Fitzgerald, Samuel H. Zingales, and Ramon DePaula, "Overview of the Mars Laser Communications Demonstration Project", AIAA Space Conference, paper 2003-6417, 2003.

2. D. M. Borosn, A. Biswas, and B. L. Edwards, "MLCD:Overview of NASA's Mars Laser Communications Demonstration System", SPIE Proceedings, vol 5338: Free Space Laser Communication Technologies XVI, pp. 16-23, January 27, 2004. 